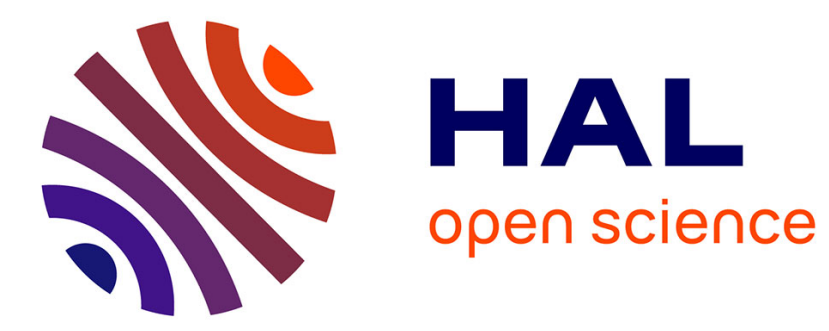

\title{
Une comparaison internationale des perceptions des paysages forestiers
}

\author{
Jean Bouchon
}

\section{To cite this version:}

Jean Bouchon. Une comparaison internationale des perceptions des paysages forestiers. Revue forestière française, 1984, 36 (1), pp.71-73. 10.4267/2042/21710 . hal-03423800

\section{HAL Id: hal-03423800 https://hal.science/hal-03423800}

Submitted on 10 Nov 2021

HAL is a multi-disciplinary open access archive for the deposit and dissemination of scientific research documents, whether they are published or not. The documents may come from teaching and research institutions in France or abroad, or from public or private research centers.
L'archive ouverte pluridisciplinaire HAL, est destinée au dépôt et à la diffusion de documents scientifiques de niveau recherche, publiés ou non, émanant des établissements d'enseignement et de recherche français ou étrangers, des laboratoires publics ou privés. 


\title{
nature. \\ loisirs et forêt
}

\section{UNE COMPARAISON INTERNATIONALE DES PERCEPTIONS DES PAYSAGES FORESTIERS}

\author{
J. BOUCHON
}

La fondation TOYOTA, dans le cadre des crédits qu'elle affecte à la recherche sur l'environnement, a commandé à un groupe de chercheurs japonais une étude sur la perception des paysages forestiers. Compte tenu des relations privilégiées entre ces chercheurs japonais et les chercheurs allemands et français, l'étude a été prolongée en Europe et a conduit à cette comparaison internationale.

\section{SITUATION DES VILLES OÙ L'ENQUÊTE A ÉTÉ RÉALISÉE}

6 villes japonaises

TOKYO : 8510000 habitants; beaucoup d'espaces verts et de belles forêts à proximité.

ASAHIKAWA : 349000 habitants; la deuxième ville d'Hokkaïdo; grandes forêts avoisinantes ; climat continental.

TSURUOKA: 101000 habitants; dans le nord-ouest d'Honshu; grandes forêts dans le voisinage. KUSHIBIKI: 9000 habitants; région agricole près de TSURUOKA.

INA : 55000 habitants; enchassée en forêt dans l'île de Honshu.

MIYAZAKI : 259000 habitants; sud-est de Kyushu. Climat doux.

\section{4 villes allemandes}

FRIBOURG en BRISGAU : 177000 habitants ; à proximité de la Forêt-Noire ; situation climatique et forestière analogue à celle de NANCY et d'ASAHIKAWA. 


\section{J. BOUCHON}

NEUENBÜRG : 7000 habitants ; au nord de la Forêt-Noire.

GÖTTINGEN : 257000 habitants ; ville universitaire et commerciale.

HANOVRE : 536000 habitants; forêts de résineux.

\section{1 ville française}

NANCY : 100000 habitants ; 280000 habitants dans l'agglomération.

\section{ENVIRONNEMENT FORESTIER}

Des études préliminaires sur l'environnement forestier des 11 villes ont conduit aux observations suivantes:

- Au Japon, les forêts sont situées dans les montagnes, sont difficiles d'accès et il est même le plus souvent interdit d'y pénétrer. En Europe, beaucoup de forêts sont en basse montagne ou en plaine et faciles d'accès.

- La nature, la montagne, la forêt, ont une place importante dans la culture et le sentiment religieux des Japonais; ce n'est pas le cas en Europe.

- Mise à part l'île d'Hokkaïdo, plus continentale que les autres îles, le Japon a un climat plus doux et plus humide que l'Europe.

- Les taux de boisement des trois pays sont respectivement de $25 \%, 30 \%$ et $70 \%$ pour la France, l'Allemagne et le Japon, ce qui conduit à des surfaces forestières par habitant à peu près équivalentes.

\section{DÉROULEMENT DE L'ENQUÉTE}

Les questions portaient principalement sur les sujets suivants :

- émotion, respect, sentiments religieux, perception de la forêt ; connaissance des arbres et de la forêt; loisirs en forêt; avis sur les interventions sylvicoles; goût pour tel ou tel type de forêt (comparaison de photographies).

La recherche s'est déroulée de 1978 à 1980. Dans chaque ville, un échantillon représentatif a été établi (âge, sexe, quartier...). Dans les 10 villes japonaises et allemandes, le questionnaire a été envoyé par la poste, ce qui a conduit à un taux de réponse variant de 32 à $83 \%$. A Nancy, l'enquête a été faite par interview, avec notre collaboration.

\section{RÉSULTATS}

- L'émotion ressentie par un Européen lorsqu'il entre en forêt semble plus profonde que celle d'un Japonais, notamment d'un habitant de Tokyo.

- A la question: "Où allez-vous de préférence lorsque vous faites un voyage (touristique) ?", plus de $50 \%$ des Allemands répondent en forêt, $25 \%$ des Nancéiens répondent en forêt et les 25 autres répondent la plage, tandis qu'un quart des Japonais déclare visiter des vieux temples, un cinquième déclare vouloir voir un lac, et un tiers une montagne pour les paysages qu'on y trouve.

- Les Européens citent des arbres forestiers lorsqu'on leur demande de citer des noms d'arbres : les Japonais citent des arbres d'ornement ; l'éventail des espèces citées est beaucoup plus ouvert au Japon : cela peut être dû à la relative pauvreté de la flore européenne. 
- Les Allemands préfèrent à $80 \%$ la nature modelée par l'homme; $70 \%$ des Nancéiens préfèrent la nature originelle. Les Japonais se situent à peu près à mi-chemin entre Allemands et Nancéiens, sauf Tokyo, assez proche de Nancy. Par contre, les Nancéiens, comme les Allemands, déclarent qu'il faut intervenir pour " maintenir " les forêts propres et belles.

\section{CRITIQUES ET CONCLUSIONS}

L'analyse des résultats montre que Nancy se distingue assez nettement des deux autres pays. Les Nancéiens ont répondu comme les Allemands pour certaines questions, mais comme les Japonais de TOKYO ou de MIYAZAKI pour d'autres; cette position intermédiaire est difficile à interpréter ; elle peut être due au fait qu'une seule ville a été sondée en France et que cette ville n'est peut-être pas représentative; elle peut aussi être due à la différence de technique de sondage: à Nancy, la quasi-totalité des personnes choisies a priori comme échantillon ont répondu (technique de l'interview). Dans les deux autres pays, l'envoi par la poste ne rapporte que les réponses des personnes intéressées par l'enquête, ce qui discrimine la population des personnes qui répondent de la population échantillonnée a priori.

Une autre critique vient du fait qu'il a été difficile de traduire certains concepts. Ainsi la question : "Avez-vous déjà eu l'impression que dans bien des choses de la nature, comme montagnes et vallées, fleuves et ruisseaux, arbres et plantes, habite quelque chose qui ressemble à une âme ? " n'a certainement pas eu d'équivalents exacts dans les trois langues.

Ainsi encore la question française : "Pensez-vous que la chasse est un bon sport? " a été traduite en allemand: "Pensez-vous que la chasse est une bonne chose?".

D'autre part, même pour une question dont la traduction ne présentait pas de difficultés, l'interprétation pose des problèmes. Ainsi la question: "Où allez-vous de préférence lorsque vous faites un voyage? " était suivie d'un certain nombre d'options telles que forêt, vieilles églises, etc. Mais, il faut savoir que d'une part les nombres de journées de vacances au Japon et en Europe sont très différents et, d'autre part, qu'au Japon, les vieilles églises sont presque toujours dans un cadre forestier ou des parcs, alors qu'en Europe, elles sont souvent au cœur d'agglomérations. Les connotations des mots "voyages" ou "vieilles églises" sont donc probablement très différentes au Japon et en Europe.

On peut dire que cette enquête montre d'assez nettes différences entre les trois pays, mais qu'il est parfois difficile d'en conclure que ces différences sont dues aux populations échantillonnées ou à des artefacts dus à l'enquête.

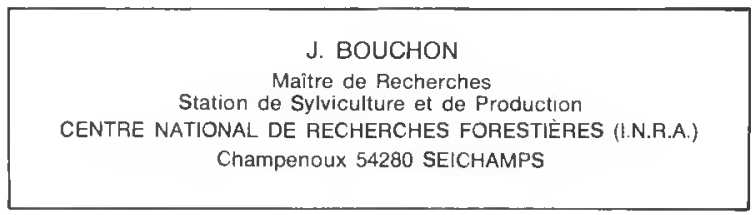

\section{BIBLIOGRAPHIE}

SHIDEI Tsunahide. - International Comparisons of Attitudes toward Nature. (The Institute of Statistical Mathematics, 4-6-7 Minamiazabu Minatoku, Tokyo, 106, Japon). Septembre 1981, 140 pages. 